\title{
Mitochondrial reactive oxygen species and complex II levels are associated with the outcome of hepatocellular carcinoma
}

\author{
JIANHUA WU $^{1}$, FEI ZHAO ${ }^{2}$, YUFEI ZHAO ${ }^{2}$ and ZHANJUN GUO ${ }^{2}$ \\ ${ }^{1}$ Experimental Animal Center; ${ }^{2}$ Department of Gastroenterology and Hepatology, \\ The Fourth Hospital of Hebei Medical University, Shijiazhuang, Hebei 050011, P.R. China
}

Received October 20, 2014; Accepted July 28, 2015

DOI: $10.3892 / \mathrm{ol} .2015 .3621$

\begin{abstract}
In the present study, two oxidative stress parameters, reactive oxygen species (ROS) and mitochondrial respiratory complex II, were evaluated in the mitochondria of hepatocellular carcinoma (HCC) cells to determine the association between these parameters and the carcinogenesis and clinical outcome of HCC. High levels of ROS and low levels of complex II were found to be associated with reduced post-operative survival in HCC patients using the log-rank test. Furthermore, multivariate analysis confirmed that the levels of ROS [relative risk $(\mathrm{RR})=2.867 ; 95 \%$ confidence interval $(\mathrm{CI})$, 1.062-7.737; $\mathrm{P}=0.038]$ and complex II $(\mathrm{RR}=5.422 ; 95 \% \mathrm{CI}$, 1.273-23.088; $\mathrm{P}=0.022$ ) were independent predictors for the survival of patients with HCC. Therefore, the analysis of ROS and complex II levels may provide a useful research and therapeutic tool for the prediction of HCC prognosis and treatment.
\end{abstract}

\section{Introduction}

Hepatocellular carcinoma (HCC), which accounts for almost 598,000 mortalities each year, is the fifth most common type of cancer and the third leading cause of cancer-associated mortality worldwide (1). The risk factors for $\mathrm{HCC}$ include hepatitis $\mathrm{B}$ virus (HBV) or hepatitis $\mathrm{C}$ virus (HCV) infection, alcoholic liver disease and non-alcoholic fatty liver disease. Of these, HBV is the major risk factor for HCC (2). The majority of HCC cases $(>80 \%)$ occur in either sub-Saharan Africa or Eastern Asia, with China accounting for $>50 \%$ of worldwide cases, demonstrating age-standardized incidence rates of 35.2 and 13.3 cases per 100,000 men and women, respectively (3). Tumor recurrence is common following local treatments, and there continues to be no curative therapy once

Correspondence to: Dr Zhanjun Guo, Department of Gastroenterology and Hepatology, The Fourth Hospital of Hebei Medical University, 12 Jiankang Road, Shijiazhuang, Hebei 050011, P.R. China

E-mail: zjguo5886@aliyun.com

Key words: reactive oxygen species, complex II, hepatocellular carcinoma, outcome the tumor reaches an advanced stage (4). Numerous factors, including tumor size, number of lesions, cell differentiation, venous invasion and degree of inflammation, have been identified as predictors of recurrence and prognosis in patients with HCC. However, few studies have focused on the association between oxidative stress and the prognosis of HCC (5).

Reactive oxygen species (ROS) are generated as byproducts of mitochondrial respiration, and include superoxide $\left(\mathrm{O}_{2}{ }^{-}\right)$ and hydrogen peroxide $\left(\mathrm{H}_{2} \mathrm{O}_{2}\right)$. In particular, the superoxide anion $\left(\mathrm{O}_{2}^{-}\right)$mediates oxidative damage to numerous intracellular components, including proteins, nucleic acids and lipid membranes $(6,7)$. This ROS-induced damage may contribute to carcinogenesis and numerous other diseases (8). DNA damage mediated by oxidative stress and the ensuing genetic mutations are crucial for the pathogenesis and progression of certain human malignancies $(9,10)$. Mitochondrial DNA (mtDNA) is the only extra-nuclear genetic material in mammalian cells that is more susceptible to DNA damage and mutation than nuclear DNA. This is due to the high levels of ROS, a lack of protective histones and a limited capacity for DNA repair $(11,12)$. Damage to mtDNA due to elevated ROS production is widely accepted as a key event in the initiation and promotion of tumorigenesis (13).

Mitochondrial complex II, also termed succinate:quinone oxidoreductase or succinate dehydrogenase, oxidizes succinate to fumarate in the Krebs cycle and reduces ubiquinone in the respiratory chain. Complex II is ubiquitous in diverse aerobic species and contributes significantly to the excessive production of ROS by mitochondria $(14,15)$. Recently, several tumors caused by complex II gene mutations have been identified (16).

Although numerous studies have suggested the value of oxidative stress markers, such as ROS and complex II (14-16), in molecular cancer research, little is known regarding the potential predictive power of oxidative stress markers for the prognosis of HCC patients. The aim of the present study was to evaluate the ability of ROS and complex II mtDNA levels in HCC liver tissues for the survival of patients with HCC.

\section{Materials and methods}

Tissue specimens and mitochondrial isolation. Histologically confirmed cancerous liver tissues were obtained from 42 patients with HBV-HCC that underwent liver cancer 
resection at the Department of Hepatobiliary Surgery of The Fourth Hospital of Hebei University (Shijiazhuang, China) between March 2007 and September 2008. All the tissues were stored in liquid nitrogen immediately following surgical resection. Mitochondria were isolated using an animal tissue mitochondria isolation kit (GenMed Scientifics, Shanghai, China) and mitochondrial protein was extracted using an animal tissue mitochondria lysis kit (GenMed Scientifics).

Ethical approval. The protocol of the present study was approved by the ethics committee of The Fourth Hospital of Hebei Medical University. Written informed consent for the collection of samples and subsequent analysis was obtained from all patients.

Measurement of mitochondrial ROS activity. A quantitative colorimetric method was adopted to evaluate the activity level of ROS, using a tissue ROS determination kit (GenMed Scientifics). All experiments were conducted according to the manufacturer's instructions. Each experiment was performed three times, and the absorbance in each well was measured at a wavelength of $420 \mathrm{~nm}$, using a Synergy 2 Multi-Mode Microplate Reader (BioTek Instruments Inc., Winooski, VT, USA). The activity was calculated using the following formula: Specimen activity $=\mathrm{OH}^{-} \mu \mathrm{M} / \mathrm{L}$.

Measurement of the mitochondrial complex II levels. A quantitative colorimetric method was used to evaluate the activity level of complex II, and a tissue complex II determination kit was used for the detection of complex II (GenMed Scientifics). All experimental steps were performed according to the manufacturer's instructions. Each sample was tested in triplicate, and the absorbance in each well was measured at a wavelength of $600 \mathrm{~nm}$ using a Synergy 2 Multi-Mode Microplate Reader (BioTek Instruments, Inc.). The activity was calculated using the following formula: Specimen activity $=\mathrm{U} / \mathrm{mg}$, where $1 \mathrm{U}=$ dichlorophenol $\mu \mathrm{M} / \mathrm{min}$ - indophenol $\mu \mathrm{M} / \mathrm{min}$.

Statistical analysis. Data were expressed as inter-quartile ranges and survival curves were constructed using the Kaplan-Meier method. Comparisons between the curves were performed using the log-rank test. Multivariate survival analysis was performed using the Cox proportional hazards model. Pearson's correlation test was used to analyze the association between the level of ROS and complex II. All statistical analyses were performed using the SPSS 18.0 software package (SPSS, Inc., Chicago, IL, USA). P $<0.05$ was considered to indicate a statistically significant difference.

\section{Results}

Clinical characteristics of patients with HCC. A total of 42 patients with HBV-HCC were enrolled in the present study. A review was conducted every 3 months for 3 years, and no patient received adjuvant chemotherapy or radiation therapy following $\mathrm{HCC}$ resection. The association between the data collected during the 3-year follow-up and the clinical characteristics of the patients was calculated using the Kaplan-Meier method and the log-rank test. Patient gender, patient age, tumor size and number of tumors were not statistically significant
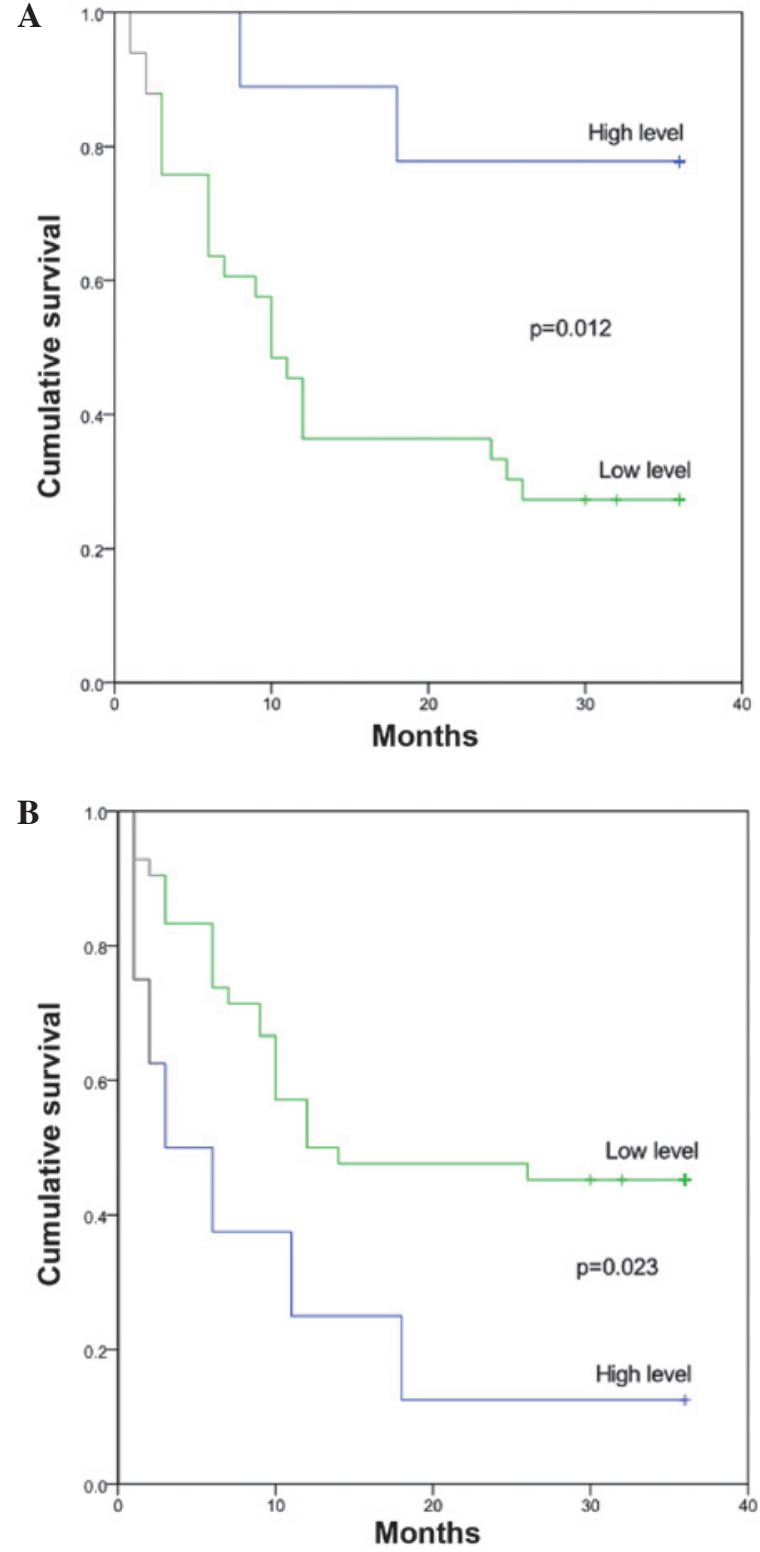

Figure 1. Survival curve according to the association between the survival rate of hepatocellular carcinoma patients and the activity of (A) complex II and $(B)$ reactive oxygen species.

predictors for the post-operative survival time. However, Child-Pugh classification and portal vein thrombosis were significantly associated with the survival time (Table I).

Association between the oxidative stress parameters and HCC outcome. The association between ROS and complex II and the outcome of HCC was assessed using the Kaplan-Meier method. The HCC patients were divided into two groups, consisting of the upper quartile and lower three quartiles, based on the ROS and complex II levels. High levels of ROS and low levels of complex II were associated with a shorter survival time, as assessed by the log-rank test $(\mathrm{P}=0.017$ and $\mathrm{P}=0.04$ for ROS and complex II, respectively) (Table II; Fig. 1). However, the levels of ROS and complex II were not correlated when assessed by linear correlation analysis $(\mathrm{r}=-0.110, \mathrm{P}=0.489)$.

Multivariate analysis was performed to determine the outcome predictors, which consisted of the Child-Pugh 
Table I. Multivariate analysis of prognostic factors associated with post-operative survival in hepatocellular carcinoma patients, performed using the Cox proportional hazards model.

\begin{tabular}{lccr}
\hline Factors & Relative risk & $95 \%$ CI & P-value \\
\hline Child-Pugh classification & 16.428 & $1.632-165.376$ & 0.018 \\
Portal vein thrombosis & 4.209 & $1.519-11.661$ & 0.006 \\
Complex II activity & 5.422 & $1.273-23.088$ & 0.022 \\
ROS activity & 2.867 & $1.062-7.737$ & 0.038 \\
\hline
\end{tabular}

ROS, reactive oxygen species; CI, confidence interval.

Table II. Univariate analysis of the clinical characteristics associated with post-operative survival in hepatocellular carcinoma patients, performed using the log-rank test.

\begin{tabular}{|c|c|c|c|}
\hline Characteristics & Patients, $\mathrm{n}$ & 3 -year survival rate, $\%$ & P-value \\
\hline Gender & & & 0.769 \\
\hline Male & 36 & 33.3 & \\
\hline Female & 6 & 33.3 & \\
\hline Age & & & 0.629 \\
\hline$\leq 55$ years & 23 & 30.4 & \\
\hline$>55$ years & 19 & 36.8 & \\
\hline Tumor diameter & & & 0.165 \\
\hline$\leq 5 \mathrm{~cm}$ & 12 & 50.0 & \\
\hline$>5 \mathrm{~cm}$ & 30 & 26.7 & \\
\hline Number of tumors & & & 0.693 \\
\hline Single & 35 & 34.3 & \\
\hline Multiple & 7 & 28.6 & \\
\hline Child-Pugh classification & & & 0.003 \\
\hline A & 41 & 34.1 & \\
\hline $\mathrm{B}$ & 1 & 0.0 & \\
\hline Portal vein thrombosis & & & 0.002 \\
\hline Yes & 6 & 0.0 & \\
\hline No & 36 & 38.9 & \\
\hline TNM classification & & & 0.328 \\
\hline I-II & 20 & 40.0 & \\
\hline III & 22 & 27.3 & \\
\hline Complex II activity & & & 0.017 \\
\hline High & 11 & 77.8 & \\
\hline Low & 31 & 21.2 & \\
\hline ROS activity & & & 0.040 \\
\hline High & 11 & 16.7 & \\
\hline Low & 31 & 40.9 & \\
\hline
\end{tabular}

ROS, reactive oxygen species; TNM, tumor-node-metastasis.

classification, portal vein thrombosis, and complex II and ROS activity, using a Cox proportional hazards model. The multivariate analysis identified complex II $(\mathrm{RR}=5.422 ; 95 \%$ $\mathrm{CI}, 1.062-7.737 ; \mathrm{P}=0.022)$ and $\mathrm{ROS}(\mathrm{RR}=2.867 ; 95 \% \mathrm{CI}$, 1.273-23.088; $\mathrm{P}=0.038)$ as independent predictors for the outcome of HCC (Table I).

\section{Discussion}

Oxidative stress causes notable damage to mtDNA, which is considered to significantly contribute to carcinogenesis (17). The present study aimed to identify effective predictors of HCC prognosis by examining two parameters of oxidative 
stress. The present data provided initial support for the conclusion that the levels of complex II and ROS were associated with carcinogenesis and the outcome of HCC, using a small sample size.

A previous study has demonstrated that oxidative stress of mtDNA is associated with the post-operative survival of patients with HCC (18). In the present study, the results demonstrated that ROS levels were associated with the carcinogenesis of HCC and the post-operative survival of HCC patients. Endogenous ROS escape from the electron transport chain during the oxidative phosphorylation process, rendering nuclear and mtDNA susceptible to damage. DNA mutations resulting from this damage may promote the malignant transformation of cells (19). A certain amount of ROS is required for cellular survival and adaptation (19), which may explain to some degree the high ROS levels associated with the poor prognosis of HCC patients. Furthermore, previous studies suggest that oxidative DNA damage is associated with cancer progression, including cell proliferation, apoptosis, genetic instability and chemo-resistant phenotypes (20-22).

To the best of our knowledge, the present study is the first to report an association between complex II levels and the outcome of HCC. However, the detailed mechanism of the effect of complex II on HCC progression remains to be elucidated. Complex II contributes significantly to the production of ROS $(14,15)$, which may explain the strong association between complex II and HCC development. The levels of complex II and ROS demonstrated no association in the present analysis, suggesting that an alternative mechanism may regulate the generation of ROS in patients with HCC. Mitochondrial complex II has previously been used as a novel target for anticancer agents, and several anticancer agents may induce cancer cell apoptosis by interacting with subunits of complex II (23). Cancer cells with the highest levels of complex II were targeted and driven to apoptosis $(23,24)$.

In conclusion, the levels of ROS and complex II were identified as independent prognostic markers for the outcome of HCC. The analysis of ROS and complex II levels may provide a useful research and therapeutic tool for ROS- and complex II-associated hepatocarcinogenesis.

\section{Acknowledgements}

This study was supported by the Key Basic Research Program of Hebei (grant no. 14967713D).

\section{References}

1. Parkin DM, Bray F, Ferlay J and Pisani P: Global cancer statistics, 2002. CA Cancer J Clin 55: 74-108, 2005.

2. El-Serag HB: Hepatocellular carcinoma. N Engl J Med 365: 1118-1127, 2011.

3. El-Serag HB and Rudolph KL: Hepatocellular carcinoma: Epidemiology and molecular carcinogenesis. Gastroenterology 132: 2557-2576, 2007.

4. Hoshida Y, Villanueva A and Llovet JM: Molecular profiling to predict hepatocellular carcinoma outcome. Expert Rey Gastroenterol Hepatol 3: 101-103, 2009.

5. Okada S, Shimada K, Yamamoto J, Takayama T, Kosuge T, Yamasaki S, Sakamoto M and Hirohashi S: Predictive factors for postoperative recurrence of hepatocellular carcinoma. Gastroenterology 106: 1618-1624, 1994.
6. Csiszar A, Podlutsky A, Podlutskaya N, Sonntag WE, Merlin SZ, Philipp EE, Doyle K, Davila A, Recchia FA, Ballabh $\mathrm{P}$, et al: Testing the oxidative stress hypothesis of aging in primate fibroblasts: Is there a correlation between species longevity and cellular ROS production? J Gerontol A Biol Sci Med Sci 67: 841-852, 2012.

7. Park J, Lee J and Choi C: Mitochondrial network determines intracellular ROS dynamics and sensitivity to oxidative stress through switching inter-mitochondrial messengers. PLoS One 6: e23211, 2011.

8. Kawanishi S, Hiraku Y and Oikawa S: Mechanism of guanine-specific DNA damage by oxidative stress and its role in carcinogenesis and aging. Mutat Res 488: 65-76, 2001.

9. Valko M, Leibfritz D, Moncol J, Cronin MT, Mazur M and Telser J: Free radicals and antioxidants in normal physiological functions and human disease. Int J Biochem Cell Biol 39: 44-84, 2007.

10. DeNicola GM, Karreth FA, Humpton TJ, Gopinathan A, Wei C, Frese K, Mangal D, Yu KH, Yeo CJ, Calhoun ES, et al: Oncogene-induced Nrf2 transcription promotes ROS detoxification and tumorigenesis. Nature 475: 106-109, 2011.

11. DiMauro S and Schon EA: Mitochondrial DNA mutations in human disease. Am J Med Genet 106: 18-26. 2001.

12. Lightowlers RN, Chinnery PF, Turnbull DM and Howell N: Mammalian mitochondrial genetics: Heredity, heteroplasmy and disease. Trends Genet 13: 450-455, 1997.

13. Yang L, Wang P, Wang H, Li Q, Teng H, Liu Z, Yang W, Hou L and Zou X: Fucoidan derived from Undaria pinnatifida induces apoptosis in human hepatocellular carcinoma SMMC-7721 cells via the ROS-mediated mitochondrial pathway. Mar Drugs 11:1961-1976, 2013.

14. Quinlan CL, Orr AL, Perevoshchikova IV, Treberg JR, Ackrell BA and Brand MD: Mitochondrial complex II can generate reactive oxygen species at high rates in both the forward and reverse reactions. J Biol Chem 287: 27255-27264, 2012

15. Moreno-Sánchez R, Hernández-Esquivel L, Rivero-Segura NA, Marín-Hernández A,Neuzil J, Ralph SJ and RodríguezEnríquez S: Reactive oxygen species are generated by the respiratory complex II - evidence for lack of contribution of the reverse electron flow in complex I. FEBS J 280: 927-938, 2013.

16. Hoekstra AS and Bayley JP: The role of complex II in disease. Biochim Biophys Acta 1827: 543-551, 2013.

17. Halliwell B: Can oxidative DNA damage be used as a biomarker of cancer risk in humans? Problems, resolutions and preliminary results from nutritional supplementation studies. Free Radic Res 29: 469-486, 1998.

18. Li S, Wang X, Wu Y, Zhang H, Zhang L, Wang C, Zhang R and Guo Z: 8-Hydroxy-2'-deoxyguanosine expression predicts hepatocellular carcinoma outcome. Oncol Lett 3: 338-342, 2012.

19. Cheng CW, Kuo CY, Fan CC, Fang WC, Jiang SS, Lo YK, Wang TY, Kao MC and Lee AY: Overexpression of Lon contributes to survival and aggressive phenotype of cancer cells through mitochondrial complex I-mediated generation of reactive oxygen species. Cell Death Dis 4: e681, 2013.

20. Inoue $S$ and Kawanishi S: Oxidative DNA damage induced by simultaneous generation of nitric oxide and superoxide. FEBS Lett 371: 86-88, 1995.

21. Erhola M, Toyokuni S, Okada K, Tanaka T, Hiai H, Ochi H, Uchida K, Osawa T, Nieminen MM, Alho H, et al: Biomarker evidence of DNA oxidation in lung cancer patients: Association of urinary 8-hydroxy-2'-deoxyguanosine excretion with radiotherapy, chemotherapy, and response to treatment. FEBS Lett 409: 287-291, 1997.

22. Miyake H, Hara I, Gleave ME and Eto H: Protection of androgen-dependent human prostate cancer cells from oxidative stress-induced DNA damage by overexpression of clusterin and its modulation by androgen. Prostate 61: 318-323, 2004.

23. Kluckova K, Bezawork-Geleta A, Rohlena J, Dong L and Neuzil J: Mitochondrial complex II, a novel target for anti-cancer agents. Biochim Biophys Acta 1827: 552-564, 2013.

24. Albayrak T, Scherhammer V, Schoenfeld N, Braziulis E, Mund T, Bauer MK, Scheffler IE and Grimm S: The tumor suppressor cybL, a component of the respiratory chain, mediates apoptosis induction. Mol Biol Cell 14: 3082-3096, 2003. 